Cite this: Phys. Chem. Chem. Phys., 2014, 16, 3846

Received 27th September 2013, Accepted 17th December 2013

DOI: $10.1039 / c 3 c p 54103 g$

www.rsc.org/pccp

\title{
X-ray absorption spectroscopy and resonant inelastic scattering study of the first lithiation cycle of the Li-ion battery cathode $\mathrm{Li}_{2-x} \mathrm{MnSiO}_{4}$
}

\author{
P. T. Kristiansen, ${ }^{a b c}$ M. Dahbi, ${ }^{d}$ T. Gustafsson, ${ }^{d}$ K. Edström, ${ }^{d}$ D. Newby, ${ }^{e}$ K. E. Smith ${ }^{e}$ \\ and L.-C. Duda*a
}

\begin{abstract}
We employ soft X-ray absorption spectroscopy and resonant inelastic X-ray scattering spectroscopy to study the redox behavior in the first lithiation/delithiation cycle of $\mathrm{Li}_{2-x} \mathrm{MnSiO}_{4}(4.0-4.6 \mathrm{~V})$. For extraction of lithium ions up to an end potential of $4.1 \mathrm{~V}$, we do not detect any change in the oxidation state for the expected redox-active $\mathrm{Mn}$ atom, instead the electronic structure of the $\mathrm{Si}-\mathrm{O}$ network is affected. Above $4.1 \mathrm{~V}$, there is an abrupt change in the oxidation state of the $\mathrm{Mn}$-ions, from $2+$ to $4+$, which is accompanied by a complete loss of long range order in the material, as detected by $\mathrm{X}$-ray diffraction. Further lithium extraction leads to progressive loss of crystallinity of $\mathrm{Li}_{2-x} \mathrm{MnSiO}_{4}$, rather than formation of a new structure, explaining the measured first-cycle capacity loss of this material. Our results suggest that future improvement of the crystalline stability of the material, particularly with respect to the $\mathrm{SiO}_{4}$ network, is required to harness the full charge capacity of $\mathrm{Li}_{2-x} \mathrm{MnSiO}_{4}$.
\end{abstract}

\section{Introduction}

Rechargeable lithium-ion batteries, already widely in use in portable electronic devices, are now envisioned to power more demanding applications, such as electric vehicles. In order to take this step, it is crucial to further improve key properties of these rechargeable lithium-ion batteries, such as capacity, rate capability, and cycle life, among other things. To contribute to this goal, most investigations are directed at the improvement of cathode materials. ${ }^{1}$ Among the recently explored cathode materials are polyanion-based cathode materials, typically, phosphates and silicates. In particular, $\mathrm{LiFePO}_{4}{ }^{2}$ and $\mathrm{Li}_{2} \mathrm{FeSiO}_{4}{ }^{3}$ open up a new area of investigation due to their good characteristics, such as low cost, safety, and high capacity. The most striking advantage of the orthosilicate family with the general formula $\mathrm{Li}_{2} \mathrm{MSiO}_{4}(\mathrm{M}=\mathrm{Fe}, \mathrm{Mn}, \mathrm{Co})$ is the existence of two lithium ions per formula unit. ${ }^{3-9}$ The chemical formula implies that it is

\footnotetext{
${ }^{a}$ Department of Physics and Astronomy, Division of Molecular and Condensed Matter Physics, Uppsala University, Box 516, S-751 20 Uppsala, Sweden. E-mail: Laurent.Duda@physics.uu.se

${ }^{b}$ Abteilung Anorganische Chemie, Fritz-Haber-Institut der Max-Planck-Gesellschaft, Faradayweg 4-6, D-14195 Berlin, Germany

${ }^{c}$ Helmholtz-Zentrum Berlin für Materialien und Energie, Albert-Einstein-Str. 15, D-12489 Berlin, Germany

${ }^{d}$ Department of Chemistry - Ångström Laboratory, Uppsala University, Box 538, S-751 21 Uppsala, Sweden

${ }^{e}$ Department of Physics, Boston University, 590 Commenwealth Ave., Boston, MA 02215, USA
}

possible to extract two Li ions per transition metal unit, giving a very high theoretical specific capacity of about $330 \mathrm{~mA} \mathrm{~h} \mathrm{~g}{ }^{-1}$. However, both lithium ions could not be extracted from the initially proposed orthosilicate cathode material, $\mathrm{Li}_{2} \mathrm{FeSiO}_{4}$, due to usual $\mathrm{Fe}$ atom ionic states and electrolyte restrictions. ${ }^{4}$ By contrast, the second lithium can be readily removed from $\mathrm{Li}_{2} \mathrm{MnSiO}_{4}$ from the host structure (using the $\mathrm{Mn}^{3+} / \mathrm{Mn}^{4+}$ redox couple) to achieve a higher capacity. ${ }^{5}$

By contrast, the electrochemical behavior of $\mathrm{Li}_{2} \mathrm{MnSiO}_{4}$ appears to be much worse than that of $\mathrm{Li}_{2} \mathrm{FeSiO}_{4} \cdot{ }^{3,10}$ Until now, only a few reports have appeared in the literature on the synthesis and characterization of $\mathrm{Li}_{2} \mathrm{MnSiO}_{4}$ either by the solid state or sol-gel route. ${ }^{5-7}$ Dominko et al. first reported the synthesis and electrochemical performance of $\mathrm{Li}_{2} \mathrm{MnSiO}_{4}$ by a modified Pechini sol-gel technique. ${ }^{5}$ In this process only $0.6 \mathrm{Li}$ per formula unit could be extracted in the first cycle and $0.3 \mathrm{Li}$ can be reversibly extracted during the fifth cycle at the $\mathrm{C} / 30$ rate. Belharouak et al. were able to achieve initial discharge capacities of about $135 \mathrm{~mA} \mathrm{~h} \mathrm{~g}^{-1}$ at room temperature, over a wide voltage range, by using carbon-coated submicron-sized particles, but this is still far less than predicted for complete extraction of all the lithium. ${ }^{7}$ At that time, the extraction of more than one electron per formula unit $\left(209 \mathrm{~mA} \mathrm{~h} \mathrm{~g}{ }^{-1}\right.$, corresponding to 1.25 electrons exchange per formula unit) was only attained on the first cycle with a large capacity loss for subsequent cycles. ${ }^{11}$ Recently, a very stable discharge behavior $\left(\sim 140 \mathrm{~mA} \mathrm{~h} \mathrm{~g}{ }^{-1}\right)$ was reported for up to 40 cycles for $\mathrm{Li}_{2} \mathrm{MnSiO}_{4}$ synthesized by a solid state reaction. ${ }^{12}$ Similarly, a hydrothermal route that led to a flower-like morphology showed a quite stable 
capacity of $100 \mathrm{~mA} \mathrm{~h} \mathrm{~g}{ }^{-1} \cdot{ }^{13}$ It has also been reported that the nature of synthesized polymorphs can strongly influence the electrochemical behavior of $\mathrm{Li}_{2} \mathrm{MnSiO}_{4}$. The materials in all studies mentioned above have an orthorhombic structure isostructural with orthorhombic $\mathrm{Li}_{2} \mathrm{FeSiO}_{4}$ (Pmn21 space group), known as the $\beta_{\text {II }}$ phase. The crystal structure of its high-temperature form $(P 21 / n)$ has been reported by Politaev et al., which is similar to that of monoclinic $\mathrm{Li}_{2} \mathrm{FeSiO}_{4}$, where only $4 \% \mathrm{Li}$ could be extracted in the usual voltage range. ${ }^{14}$ Additionally, two other polymorphs have also been synthesized recently with orthorhombic (Pmnb) and monoclinic $(P n)$ structures. ${ }^{15,16}$

Note that in all these investigations the large irreversible capacity between charge and discharge in the first cycle was higher than that measured in subsequent cycles. This large irreversible capacity of the first cycle is not fully understood. Most of the previous studies have tentatively ascribed this fading to both the poor electronic properties of the material and the likely structural collapse. For example, in situ XRD experiments showed that the structure of $\mathrm{Li}_{2} \mathrm{MnSiO}_{4}$ collapsed and turned into an amorphous state upon cycling. ${ }^{11,17,18}$ This large difference between electrochemical performances of $\mathrm{Li}_{2} \mathrm{MnSiO}_{4}$ and $\mathrm{Li}_{2} \mathrm{FeSiO}_{4}$ has mainly been attributed to the Jahn-Teller distortion associated with the $\mathrm{Mn}^{3+}$, which causes a large change in the lattice parameters and destroys the crystalline structure of the material. Suppression of Jahn-Teller distortions has shown to improve the capacity retention. ${ }^{19}$ Furthermore, an in situ XAS study at the transition metal K-edges showed that such high charging capacity in $\mathrm{Li}_{2} \mathrm{MnSiO}_{4}$ material obtained by standard Pechini synthesis is not connected with the $\mathrm{Mn}^{4+}$ state. ${ }^{20}$ They showed that only $60 \%$ of $\mathrm{Mn}^{2+}$ was oxidized to $\mathrm{Mn}^{3+}$ during the charging process, implying that the probability of extracting more than one electron per formula unit was remote.

In this paper, we present a study of the first charge-discharge cycle for the cathode material $\mathrm{Li}_{2} \mathrm{MnSiO}_{4}$, in order to achieve an in-depth understanding of the origin of its capacity loss upon cycling. We apply a powerful combination of ex situ techniques: X-ray diffraction (XRD), soft X-ray absorption spectroscopy (XAS) and resonant inelastic X-ray scattering (RIXS). We also report a new combustion method for the synthesis of $\mathrm{Li}_{2} \mathrm{MnSiO}_{4}$ material by using sucrose as the carbon source. The advantages of this technique are that it is time saving, cost-effective and also allows for mass-scale production, which is a primary concern for commercial applications of Li-ion batteries. ${ }^{10}$ We show here also for the first time charge-discharge characteristics of the redox processes for the $\mathrm{Li}_{2} \mathrm{MnSiO}_{4}$ material and, by using XAS and RIXS techniques, confirm the presence of $\mathrm{Mn}^{4+}$ ions upon charging of the material.

\section{Experimental}

\section{A. Sample preparation}

The starting material $\mathrm{Li}_{2} \mathrm{MnSiO}_{4}$ was prepared by a method similar to that reported previously. ${ }^{26}$ The $\mathrm{Li}_{2-x} \mathrm{MnSiO}_{4}$ material was obtained by the combustion method using $\mathrm{LiNO}_{3}$ (Sigma-Aldrich) and $\mathrm{Mn}\left(\mathrm{NO}_{3}\right)_{2} \cdot 4 \mathrm{H}_{2} \mathrm{O}$ (Sigma-Aldrich) as the oxidant precursors, sucrose (Sigma) as fuel and $\mathrm{SiO}_{2}$ nanoparticles (Sigma). Stoichiometric amounts of reagent grade $\mathrm{Li}, \mathrm{Mn}$ and $\mathrm{Si}$ sources were dissolved in a minimum amount of distilled water; the fuel (sucrose) was then added to the solution. The beaker containing the reaction mixture was placed on an electric heater and kept at $120{ }^{\circ} \mathrm{C}$ for 2 hours to evaporate the excess water. The liquid adopted a syrup consistency and the colour changed from white to green while the syrup swelled up and transformed into dark green foam. Upon continuing heating, this foamy mass started to burn spontaneously without flame and transformed finally into light and downy black powder. The as-formed powder was collected, ground in an agate mortar, and further heat-treated at $600{ }^{\circ} \mathrm{C}$ for $10 \mathrm{~h}$ under an argon atmosphere.

Positive electrodes were prepared by spreading a mixture of $75 \%$ active material, $15 \%$ carbon black, and $10 \%$ of PVDF [poly(vinylidene fluoride)] dissolved in NMP (1-methyl2pyrrolidinone) onto an aluminium foil. Circular electrodes (area: $3.14 \mathrm{~cm}^{2}$ ) were dried under vacuum at $120{ }^{\circ} \mathrm{C}$ inside an argon-filled glove box $\left(<3\right.$ ppm $\mathrm{H}_{2} \mathrm{O}$ and $\mathrm{O}_{2}$ ) before cell assembly. Batteries comprising the dried positive electrode, a glass fibre separator soaked in electrolyte, and a lithium metal counter electrode ( $0.38 \mathrm{~mm}$ thick) were assembled and packed in the polymer-coated aluminium pouch in a so called "coffee bag" configuration. ${ }^{27}$ The electrolyte was $1 \mathrm{M} \mathrm{LiPF}_{6}$ (Tomyama, dried overnight at $80{ }^{\circ} \mathrm{C}$ in a vacuum furnace in the glove box) in an EC/DEC (Merck, battery grade and used as received) 2:1 by volume mixture.

We assembled a series of identical batteries containing a $\mathrm{Li}_{2-x} \mathrm{MnSiO}_{4}$ cathode according to the preparation procedure described above. After assembly, one of the battery samples was kept in its pristine state, the other battery samples were charged from their pristine state and stopped at a certain potential (4.0-4.6 V) corresponding to lithium contents in $\mathrm{Li}_{2-x} \mathrm{MnSiO}_{4}$ from $x=0$ to $x \approx 2$. One of these batteries was first charged up to a potential of $4.6 \mathrm{~V}$ and thereafter allowed to discharge again. Fig. 1 shows one complete cycling curve, with an extraction charge capacity of $305 \mathrm{~mA} \mathrm{~h} \mathrm{~g}^{-1}$ and a discharge capacity of $150 \mathrm{~mA} \mathrm{~h} \mathrm{~g}{ }^{-1}$. The circled dots lettered $a-h$ mark the potential that the corresponding sample battery has been stopped at (Fig. 2). The electrochemical cycling was performed using a Digatron BTS600 battery testing system at $60{ }^{\circ} \mathrm{C}$.

\section{B. Structural characterization by $\mathrm{X}$-ray diffraction}

X-ray diffraction (XRD) patterns of $\mathrm{Li}_{2} \mathrm{MnSiO}_{4}$ were collected on a Siemens $\mathrm{D} 5000$ diffractometer with $\mathrm{Cu} \mathrm{K}_{\alpha}$ radiation. The diffraction pattern was recorded in an angular range of $2 \theta=$ $10-100^{\circ}$, using a $\Delta(2 \theta)=0.02^{\circ}$ step and a constant counting time of $10 \mathrm{~s}$. The lattice parameters were refined by the Rietveld method using the Fullprof program. ${ }^{28}$ The diffraction patterns shown in Fig. 3 indicate that a well crystallized $\beta$ phase is obtained for $\mathrm{Li}_{2} \mathrm{MnSiO}_{4}$. This was confirmed by Rietveld refinement of the pattern, which yielded good agreement factors and unit cell parameters $(a=6.3094(2) \AA, b=5.3791(2) \AA, c=4.9688(2) \AA)$ in accord with those previously reported for the $\beta$ phase from combined X-ray and neutron refinements. ${ }^{29-31}$ The $\beta$ phase 


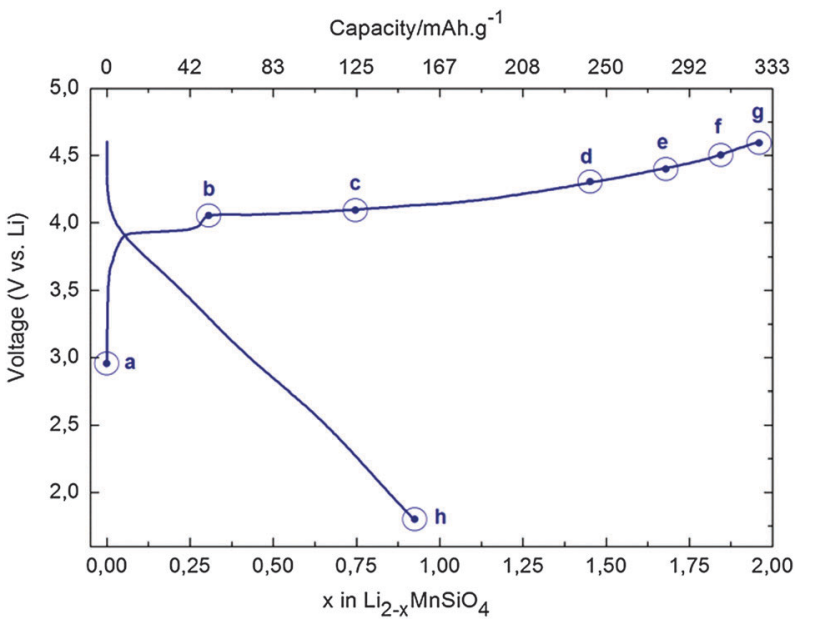

Fig. 1 Voltage profile of the $\mathrm{Li} / / \mathrm{Li}_{2-x} \mathrm{MnSiO}_{4}$ battery cell during the first charge-discharge at the $\mathrm{C} / 20$ rate. The XAS and RIXS spectra are collected at the points $(a-h)$ indicated in the voltage profile.

\begin{tabular}{|l|l|c|c|c}
\hline Cell & $\begin{array}{c}\mathrm{x} \text { in } \\
\mathrm{Li}_{2-\mathrm{x}} \mathrm{MnSiO}_{4}\end{array}$ & $\begin{array}{c}\text { Voltage } \\
\left(\mathrm{V} \text { vs. } \mathrm{Li}^{+}\right)\end{array}$ & $\begin{array}{c}\text { Curent } \\
(\mathrm{mA})\end{array}$ & $\begin{array}{c}\text { Capacity } \\
(\mathrm{mAh} / \mathrm{g})\end{array}$ \\
\hline $\mathrm{a}$ & 0 & - & - & - \\
$\mathrm{b}$ & 0.29 & 4 & 0.059 & 47 \\
$\mathrm{c}$ & 0.77 & 4.1 & 0.057 & 127 \\
$\mathrm{~d}$ & 1.45 & 4.3 & 0.053 & 241 \\
$\mathrm{e}$ & 1.67 & 4.4 & 0.051 & 278 \\
$\mathrm{f}$ & 1.85 & 4.5 & 0.057 & 305 \\
$\mathrm{~g}$ & 1.96 & 4.6 & 0.061 & 325 \\
$\mathrm{~h}$ & 0.9 & 1.8 & 0.058 & 150 \\
\hline
\end{tabular}

Fig. 2 Table with cycle data ( $x$, voltage (i.e. end potential), current, capacity) for all investigated cells (a-h).

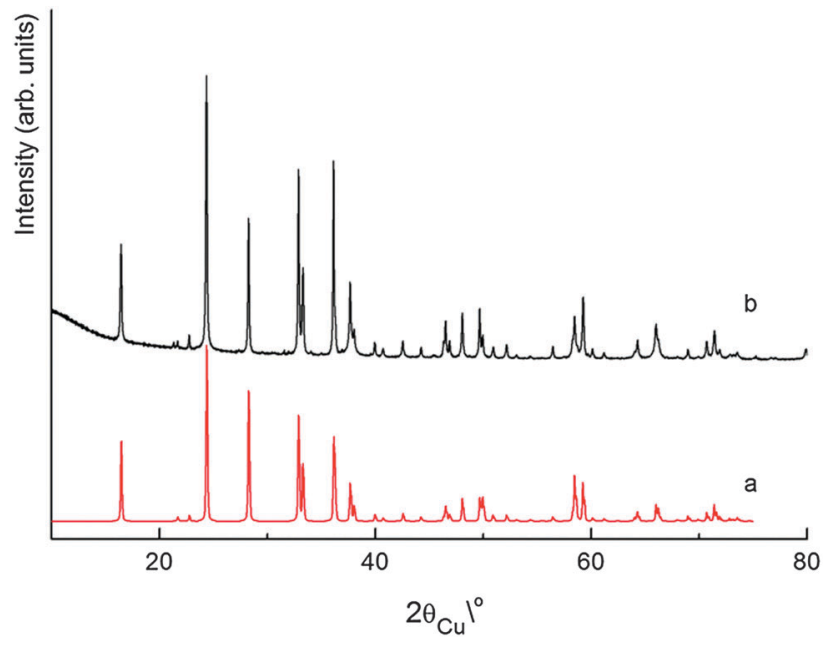

Fig. 3 (a) $\mathrm{Li}_{2} \mathrm{MnSiO}_{4}$ (JCPDS card no. 04-014-1657); (b) $\mathrm{Li}_{2-x} \mathrm{MnSiO}_{4}$ material prepared by the combustion method.

$\mathrm{Li}_{2} \mathrm{MnSiO}_{4}$ crystallizes in an orthorhombic structure with the space group Pmn21, in which the $\mathrm{MnO}_{4}{ }^{-}$and $\mathrm{SiO}_{4}$-tetrahedra alternate and connect to form layers through which lithium ions can diffuse (Fig. 4).

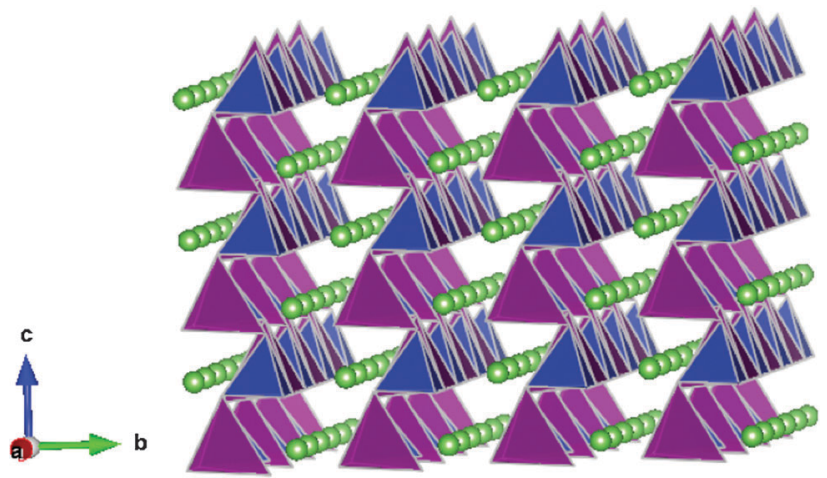

Fig. 4 Crystal structure of $\mathrm{Li}_{2} \mathrm{MnSiO}_{4}$ (pristine state). The a-axis view of a $\beta$-polymorph of $\mathrm{Li}_{2} \mathrm{MnSiO}_{4}$. The $\mathrm{SiO}_{4}$-(blue) and $\mathrm{MnO}_{4}$-(purple) tetrahedra alternate and connect to form layers through which lithium ions (green spheres) can diffuse.

On the basis of the $\mathrm{Li}_{2} \mathrm{MnSiO}_{4}$ structures, the polymorphs can be classified into low- and high-symmetry forms. All are based on a distorted hexagonal close packed array of oxide ions with all cations in distorted corner sharing tetrahedra, but they differ in the orientation of these tetrahedra, particularly for the $\mathrm{MnO}_{4}-\mathrm{SiO}_{4}$ chains. The high symmetry form (Pmn21) has $\mathrm{MnO}_{4}$ and $\mathrm{SiO}_{4}$ tetrahedra all pointing in the same direction parallel to their $c$ axes (see Fig. 4). In the lowest symmetries Pmnb and $P 21 \backslash n$ structures, half of the $\mathrm{MnO}_{4}$ and $\mathrm{SiO}_{4}$ tetrahedra point in one direction, and the other half in the opposite direction, for each of the crystals.

The $\mathrm{Li} / / \mathrm{Li}_{2} \mathrm{MnSiO}_{4}$-battery cell used for the preparation of the delithiated cathodes gives a capacity of $325 \mathrm{~mA} \mathrm{~h} \mathrm{~g}^{-1}$ on the first charge and $150 \mathrm{~mA} \mathrm{~h} \mathrm{~g}^{-1}$ on the first discharge to $1.8 \mathrm{~V}$. This initial charge capacity agrees well with extracting two lithium charges but the first discharge capacity is lower than expected. The large irreversible capacity on the first cycle could be due to effects of Jahn-Teller distortion associated with dissolution of $\mathrm{Mn}^{3+}$ or due to (partial) loss of cristallinity of $\mathrm{Li}_{2} \mathrm{MnSiO}_{4}$ at the end of the first charge. To investigate more closely the reasons for the degradation in performance of $\mathrm{Li}_{2} \mathrm{MnSiO}_{4}$, ex situ X-ray diffraction was performed for several $x$-values of $\mathrm{Li}_{2-x} \mathrm{MnSiO}_{4}$ from electrochemical cells stopped at different potentials and known lithium composition. Fig. 5 shows the selected spectra that were collected during the first charge process. The experiment shows that extraction of lithium leads to a decrease of the intensity of the main diffraction lines for the $\mathrm{Li}_{2-x} \mathrm{MnSiO}_{4}$ sample charged at $4.1 \mathrm{~V}$. At about a potential of $4.18 \mathrm{~V}(x=1.15)$ the charge curve changes its slope and at $4.3 \mathrm{~V}$ the intensity of the peaks is already so weak that it is hard to distinguish them from the background. This trend for the XRD pattern continues and there is a second change of slope at about $4.47 \mathrm{~V}(x=1.8)$ in the charge curve. The charged sample is completely amorphous and the discharge does not reverse this trend. At this point, it is not clear what causes the loss of crystallinity or at which locations significant structural changes occur in the first charge. For this reason, XAS and RIXS were used to provide valuable information related to the oxidation state of the specific atoms $(\mathrm{Mn}, \mathrm{O}, \mathrm{Si})$ as well as their 


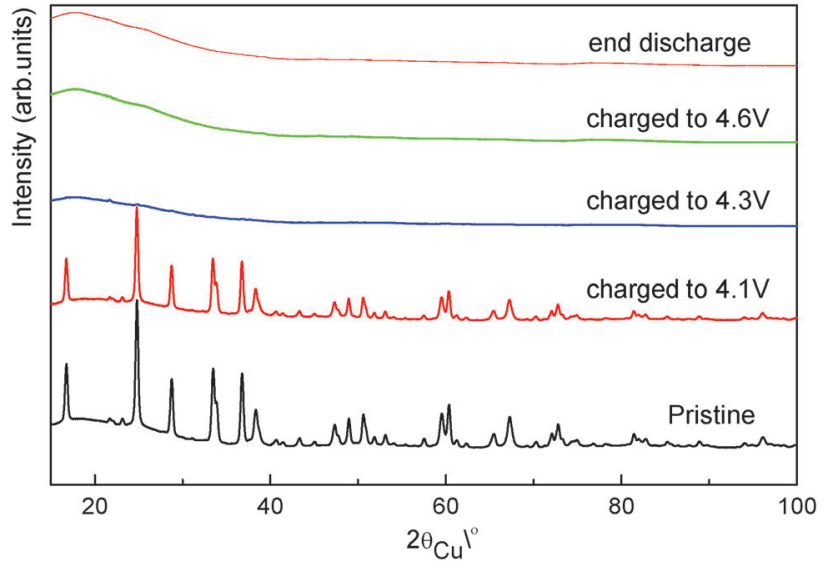

Fig. $5 \mathrm{X}$-ray diffraction patterns obtained with electrochemical oxidation of $\mathrm{Li}_{2} \mathrm{MnSiO}_{4}$-based composites at different potentials as indicated. The pristine $\mathrm{Li}_{2-x} \mathrm{MnSiO}_{4}$ pattern was inserted for easy comparison with the electrochemical delithiated $\mathrm{Li}_{2} \mathrm{MnSiO}_{4}$ patterns.

local structural environment, such as bond distances, JahnTeller distortion associated with dissolution of $\mathrm{Mn}^{3+}$.

\section{X-ray spectroscopy using synchrotron radiation}

The batteries were disassembled and the cathodes were dried in an Ar-glove box for several hours. Thereafter, a strip of each cathode was cut away and these samples were mounted adjacently on double-sided adhesive tape, vacuum sealed in an Al-pouch and transported to the synchrotron radiation facility. At the X-ray spectroscopy endstation, the tapes with the samples were mounted onto a sample holder in an $\mathrm{N}_{2}$-filled glove bag. Then the holder with the samples was removed from the glove bag and quickly transferred to a vacuum chamber and pumping commenced within less then a minute. The samples were investigated in ultra high vacuum by means of X-ray absorption spectroscopy (XAS) and resonant inelastic X-ray scattering (RIXS) ${ }^{22}$ in order to obtain site-specific electronic structure information about occupied, respectively, inoccupied states. All measurements were performed at beamline X1B. ${ }^{21}$ XAS measurements were performed at the $\mathrm{Mn} \mathrm{L}-, \mathrm{O} \mathrm{K}$ - and $\mathrm{Si}$ L-edge with beamline resolutions of $0.29 \mathrm{eV}, 0.19 \mathrm{eV}$ and $30 \mathrm{meV}$ respectively. XAS spectra using both total flourescence yield (TFY) and total electron yield (TEY) were obtained simultaneously and showed identical trends. However, for technical reasons, the statistical quality of TEY spectra was generally higher than the TFY spectra, which were therefore omitted in the following. RIXS measurements of the Mn L-edge were obtained using a Scienta XES 300 Rowland mount X-ray emission spectrometer ${ }^{22,23}$ using a bandwidth of $0.56 \mathrm{eV}$ for the incident X-rays and a spectrometer resolution of $0.52 \mathrm{eV}$.

\section{Results and discussion}

Fig. 6 shows $\mathrm{O}$ K-absorption spectra from $\mathrm{Li}_{2-x} \mathrm{MnSiO}_{4}$-cathodes at a series of end potentials, as indicated. We observe a peak in the pre-edge range between $528.0 \mathrm{eV}$ and $532.9 \mathrm{eV}$ whose

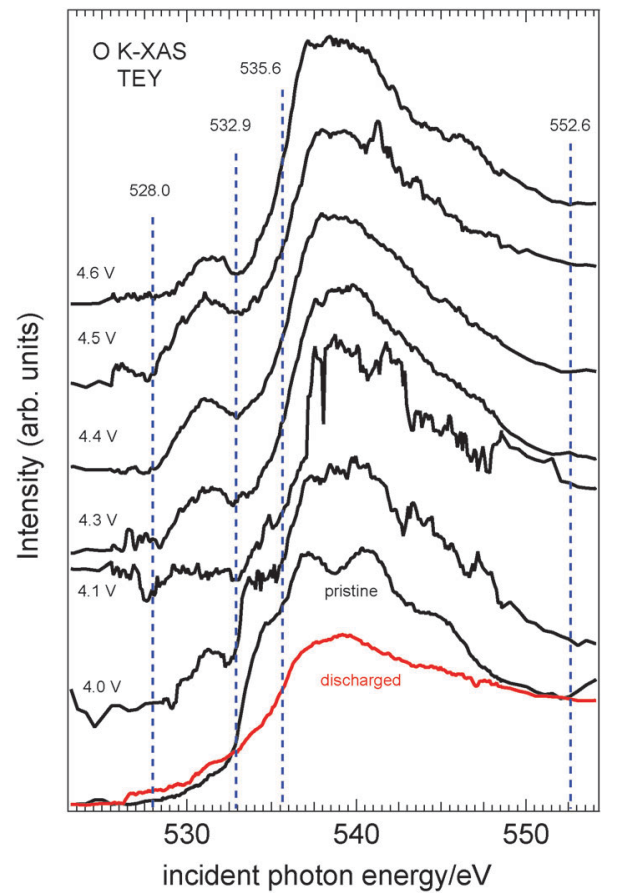

Fig. $6 \mathrm{X}$-ray absorption spectroscopy recorded in the total electron yield mode at the $\mathrm{O} \mathrm{K}$-edge of $\mathrm{Li}_{2-x} \mathrm{MnSiO}_{4}$-cathodes charged to various potentials. The dashed vertical lines (given with their energy positions in $\mathrm{eV}$ ) are guides to the eyes for distinguishing the different salient features in the spectra.

intensity varies depending on the end potential. We attribute this feature to empty $\mathrm{O} 2 \mathrm{p}$-orbitals that are hybridized with $\mathrm{Mn}$ $3 \mathrm{~d}$-states (see ref. 25 and discussion below). Another pre-edge feature is seen in the range between $532.9 \mathrm{eV}$ and $535.6 \mathrm{eV}$. This peak arises from empty $\mathrm{O} 2 \mathrm{p}$-orbitals that are hybridized with $\mathrm{Si}$ 2 p-states. Finally, there is also the main peak in the range between $535.6 \mathrm{eV}$ and $552.6 \mathrm{eV}$ that reflects $\mathrm{O} 2 \mathrm{p}$-states hybridized with delocalized s- and p-states of $\mathrm{Mn}$ and $\mathrm{Si}$.

In the pristine sample the low-energy prepeak is absent whereas the high-energy prepeak is relatively prominent. The low-energy prepeak reaches its maximum intensity between $4.4 \mathrm{~V}$ and $4.5 \mathrm{~V}$ whereas the high-energy prepeak quickly loses intensity and is almost completely obscured at $4.3 \mathrm{~V}$. Thus electrons rapidly enter the Si-derived orbitals whereas the Mn 3d-derived orbitals are first depleted and then re-filled between $4.0 \mathrm{~V}$ and $4.6 \mathrm{~V}$. The main peak has a distinct fine structure (triple-peak) that loses definition as the potential is raised. A probable explanation is the formation of defects, e.g. by $\mathrm{Si}$ atoms leaving their original tetrahedral oxygen cages (Fig. 4) (see discussion on Si L-XAS below). At $4.4 \mathrm{~V}$ the main peak shows no fine structure whereas at higher potentials the high energy side is more structured again. Finally, the spectra of the discharged cathode are superimposed on the one from the pristine. These two spectra show substantial variance in the high energy prepeak and the main peak, suggesting that the lithiation/delithiation process leads to irreversible changes in the electronic structure at the oxygen sites and in particular for the oxygens that hybridize with Si. 


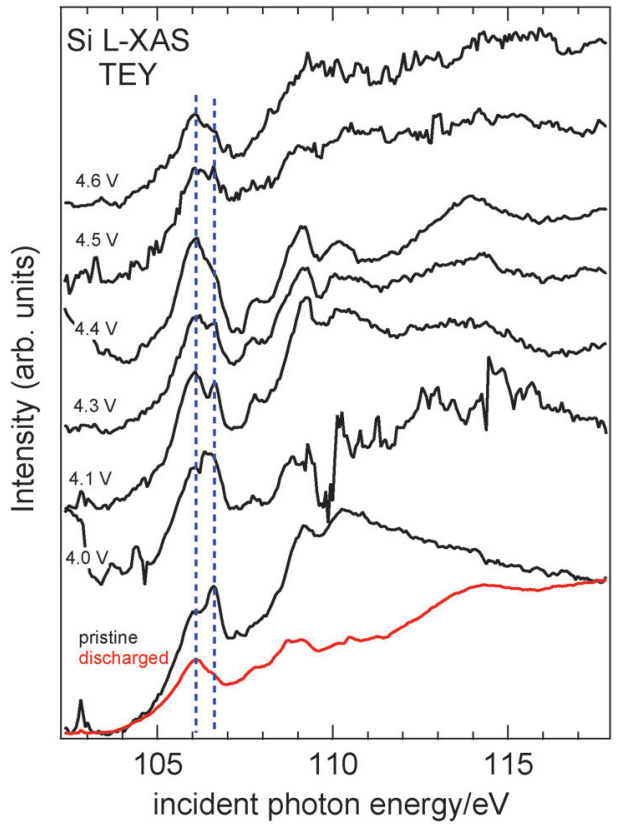

Fig. $7 \mathrm{X}$-ray absorption spectroscopy recorded in the total electron yield mode at the $\mathrm{Si} \mathrm{L}$-edge of $\mathrm{Li}_{2-x} \mathrm{MnSiO}_{4}$-cathodes for various end potentials. The vertical lines mark the positions of the two components of the first double peak.

Fig. 7 shows Si L-absorption measured from cathode samples for end potentials as above. We can discern a clear trend regarding the first double peak, namely that there is a decrease of the high energy component in this peak up to a potential of $4.4 \mathrm{~V}$. This coincides with the behavior of the second O K-XAS prepeak, suggesting that the corresponding hybridized orbitals are filled as the potential is increased. At a potential of $4.5 \mathrm{~V}$ and above there is also a marked overall decrease of intensity and a change of the spectral appearance at energies above the first double peak. This is correlated with a second change of slope of the charge curve that occurs at $4.47 \mathrm{~V}(x=1.8)$.

Fig. 8 shows Mn L-absorption measured from samples for the same end potentials as above. The pristine sample as well as the cathodes at low potentials (up to $4.1 \mathrm{~V}$ ) show a narrow $\mathrm{L}_{3}$-peak. From $4.1 \mathrm{~V}$ to $4.3 \mathrm{~V}$ a sudden change occurs, leaving a much broader $\mathrm{L}_{3}$-peak and a shift of spectral weight to higher photon energies. As the potential is further increased, there are only small changes, mostly a small increase of the low energy shoulder. Finally, the discharged cathode relapses to a narrow spectrum, resembling the spectrum of the pristine sample. Note that this trend is even clearer in the Mn L-spectra recorded in the TFY mode (not shown).

A comparison to Mn-oxides with various valances (2+ to $4+)$ suggests that the spectral shapes can be attributed to a superposition of spectra from various Mn-valences and their varying contribution for different end potentials. At low potentials there is a large weight from $\mathrm{Mn}^{2+}$ (with some $\mathrm{Mn}^{3+}$ contributions) whereas $\mathrm{Mn}^{4+}$ is seen to dominate the spectra in the potential range 4.3-4.6 V, with the largest contribution around $4.4 \mathrm{~V}$, a trend which coincides with the one of the first pre-peak of the O K-edge. When discharged, the Mn-valence relaxes back, nearly reversibly, to be dominated by a $\mathrm{Mn}^{2+}$-character again.

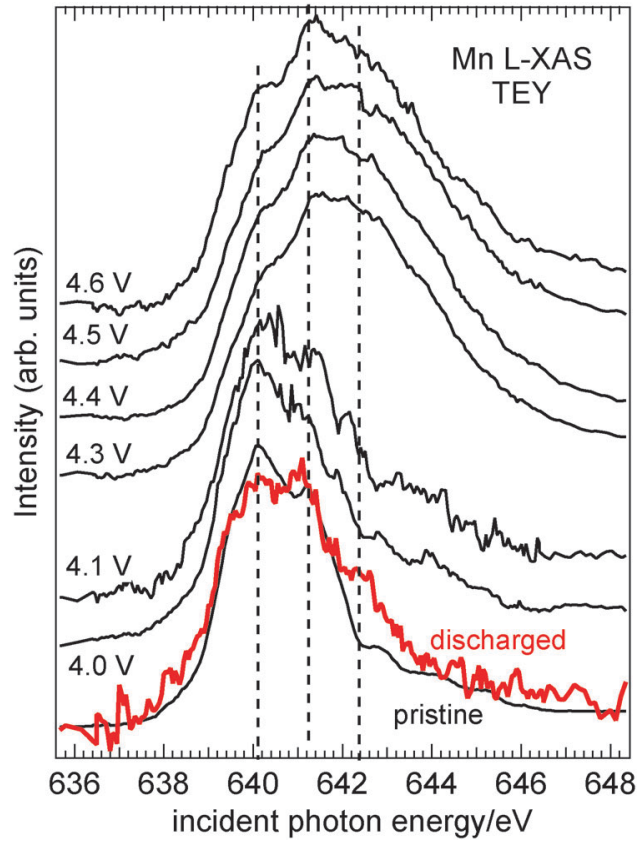

Fig. 8 X-ray absorption spectroscopy recorded in the total electron yield mode at the $\mathrm{Mn} \mathrm{L}_{3}$-edge of $\mathrm{Li}_{2-x} \mathrm{MnSiO}_{4}$-cathodes for various end potentials.

Using RIXS, excited at the lowest energy peak of the Mn L-XAS spectrum, we can project the trend for $\mathrm{Mn}^{2+}$-character very clearly. In Fig. 9a the high energy peak of the pristine cathode material corresponds to elastic scattering whereas the low energy peak $(636.5 \mathrm{eV})$ corresponds to dd-excitations from the $\mathrm{Mn} \mathrm{d}^{5}$-ground state configuration. ${ }^{24}$ The less intense low-energy region belongs to charge transfer excitations. Starting from low potentials, there is a clear break in the pattern at $4.3 \mathrm{~V}$ when the dd-excitation peak suddenly loses intensity, broadens and shifts about $+0.8 \mathrm{eV}$. Thereafter, the intensity recovers again. This corroborates clearly the pattern deduced from the Mn L-XAS.

The $+0.8 \mathrm{eV}$ shift of the dd-excitation above $4.1 \mathrm{~V}$ could suggest that the Mn tetrahedron changes structure, which in
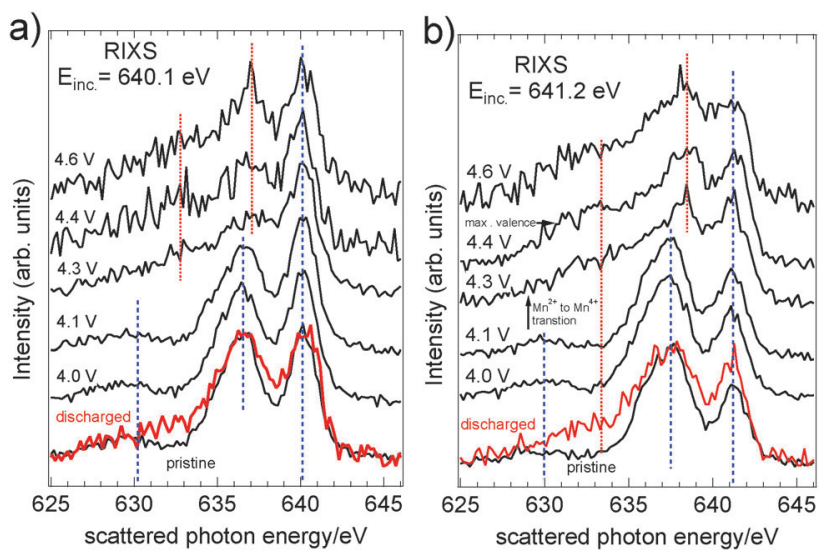

Fig. $9 \mathrm{Mn} \mathrm{L-RIXS}$ spectra of $\mathrm{Li}_{2-x} \mathrm{MnSiO}_{4}$ cathodes for various end potentials. (a) Incident energy $E_{\text {inc. }}=649.26 \mathrm{eV}$. (b) Incident energy $E_{\text {inc. }}=650.37 \mathrm{eV}$. 
turn causes the loss of the long order structure by distortions of the bonds to the Si tetrahedron, as seen by the XRD in Fig. 3. The possible change in the Mn-oxide tetrahedra appears to be reversible as the dd peaks of the pristine and discharged measurements coincide. This is not the case for the charge transfer excitations, which could indicate that the surroundings of the Mn-oxide tetrahedra may have changed during the charge cycle and that the Si-oxide tetrahedra have taken on a glass-like character.

\section{Summary and conclusion}

Using soft X-ray absorption spectroscopy and resonant inelastic $\mathrm{X}$-ray scattering spectroscopy we find that there is an abrupt valence change from predominantly $\mathrm{Mn}^{2+}$ to $\mathrm{Mn}^{4+}$ for end potentials above $4.1 \mathrm{~V}$. The manganese valence decreases again at higher potentials and in the discharged state of the battery we observe again a strong contribution from $\mathrm{Mn}^{2+}$-valence. A more gradual spectral effect of the lithiation process is observed at the oxygen sites. In particular, O K-XAS and Mn L-RIXS strongly indicate that $\mathrm{O} 2 \mathrm{p}-\mathrm{Mn} 3 \mathrm{~d}$ hybridized orbitals reach their maximum depletion at $4.4 \mathrm{~V}$, which coincides with a maximum filling of some of the Si 3s-orbitals. Another slope change of the charge curve at $4.47 \mathrm{~V}$ coincides with a more rapid change of the Si L-XAS and could point to a reorganization around the Si-sites.

XRD measurements show that the battery cathode material $\mathrm{Li}_{2-x} \mathrm{MnSiO}_{4}$ undergoes an order-to-disorder phase transition between the potentials $4.1 \mathrm{~V}$ and $4.3 \mathrm{~V}$ while the charge curve shows a change in slope around a potential of $4.18 \mathrm{~V}$. This means there is a progressive loss of crystallinity in $\mathrm{Li}_{2-x} \mathrm{MnSiO}_{4}$ during delithiation, rather than formation of a new structure. $\mathrm{X}$-ray absorption spectroscopy suggests that silicon oxide tetrahedra become defective or that their coordination is distorted upon delithiation. The driving force for this is assumed to be the oxidation of the $\mathrm{Mn}$-ions from $3+$ to $4+$, which may strongly affect the orientation of the $\mathrm{SiO}_{4}-\mathrm{MnO}_{4}$ chains in $\mathrm{Li}_{2-x} \mathrm{MnSiO}_{4}$ upon lithium delithiation.

The most striking observation is that during the first part of the charging process, up to $4.1 \mathrm{~V}$, the extraction of lithium ions is not accompanied by a change in the electronic structure of the expected redox active $\mathrm{Mn}$ atom but rather by a change of the electronic structure of the Si-O network. In the potential interval 4.1-4.3 V there is a complete oxidation of manganese to $\mathrm{Mn}^{4+}$, accompanied by a collapse of the long range order in the material. The charge extracted from the material in this potential interval is however only $0.7 \mathrm{e}^{-}$per formula unit. Thus there must be a major reorganization of the electronic structure of the material, which is also observed in the $\mathrm{Si}$ and $\mathrm{O}$ spectra. The behavior of $\mathrm{Li}_{2-x} \mathrm{MnSiO}_{4}$ during lithium extraction or insertion is found to be more complicated than that of a simple redox activity localized at the transition metal site. Note that a similar participation of the anion network in such a redox process during lithium extraction, respectively insertion, has also been observed for $\mathrm{LiMnPO}_{4}{ }^{32}$ Thus delithiation destabilizes the original $\mathrm{Li}_{2} \mathrm{MnSiO}_{4}$ structure (Pmn21) and the material has a tendency to transform into other structures (Pmnb and or $P 21 \backslash n$ ).
In conclusion, the observed primary amorphization at $4.18 \mathrm{~V}$ is mainly coupled with a change in the state of the Mn-ions and likely leads to the first-cycle capacity loss of this material. The secondary restructuring that takes place at $4.47 \mathrm{~V}$ mainly affects the Si-ions and could be an additional factor for capacity loss. Improvement of this capacity loss should therefore aim at structural stabilization of the material at high potentials, i.e. low lithium content, particularly with respect to the $\mathrm{SiO}_{4}$ network.

\section{Acknowledgements}

The Boston University contribution to this work is supported by the Department of Energy, Basic Energy Sciences, under Grant No. DE-FG02-98ER45680.

\section{References}

1 J. M. Tarascon and M. Armand, Nature, 2001, 414, 359-367.

2 A. K. Padhi, K. S. Nanjundaswamy and J. B. Goodenough, J. Electrochem. Soc., 1997, 144, 1609.

3 A. Nyten, A. Abouimrane, M. Armand, T. Gustafsson and J. O. Thomas, Electrochem. Commun., 2005, 7, 156.

4 G. Mali, C. Sirisopanaporn, C. Masquelier, D. Hanzel and R. Dominko, Chem. Mater., 2011, 23, 2735.

5 R. Dominko, M. Bele, M. Gaberscek, A. Meden, M. Remskar and J. Jamnik, Electrochem. Commun., 2006, 8(2), 217-222.

6 Z. L. Gong, Y. X. Li and Y. Yang, Electrochem. Solid-State Lett., 2006, 9, A542-A544.

7 I. Belharouak, A. Abouimrane and K. Amine, J. Phys. Chem. C, 2009, 113, 20733-20737.

8 C. Lyness, B. Delobel, A. R. Armstrong and P. G. Bruce, J. Chem. Soc., Chem. Commun., 2007, 4890-4892.

9 K. Zaghib, A. A. Salah, N. Ravet, A. Mauger, F. Gendron and C. M. Julien, J. Power Sources, 2006, 160, 1381-1386.

10 M. Dahbi, S. Urbonaita and T. Gustafsson, J. Power Sources, 2012, 205, 456-462.

11 Y. X. Li, Z. L. Gong and Y. Yang, J. Power Sources, 2007, 174, 528.

12 V. Aravindan, K. Karthikeyan, K. S. Kang, W. S. Yoon, W. S. Kimf and Y. S. Lee, J. Mater. Chem., 2011, 21, 2470.

13 V. Aravindan, K. Karthikeyan, J. W. Lee, S. Madhavi and Y. S. Lee, J. Phys. D: Appl. Phys., 2011, 44, 152001.

14 V. V. Politaev, A. A. Petrenko, V. B. Nalbandyan, B. S. Medvedev and E. S. Shvetsova, J. Solid State Chem., 2007, 180, 1045.

15 R. J. Gummow, N. Sharma, V. K. Peterson and Y. He, J. Solid State Chem., 2012, 188, 32.

16 H. Duncan, A. Kondamreddy, P. H. J. Mercier, Y. Le Page, Y. Abu-Lebdeh, M. Couillard, P. S. Whifield and I. J. Davidson, Chem. Mater., 2011, 23, 5446.

17 R. Dominko, J. Power Sources, 2008, 184, 462.

18 A. Kokalj, R. Dominko, G. Mali, A. Meden, M. Gaberscek and J. Jamnik, Chem. Mater., 2007, 19, 3633.

19 X. Li and Y. Xu, Appl. Surf. Sci., 2007, 253, 8592-8596. 
20 R. Dominko, I. Arcon, A. Kodre, D. Hanzel and M. Gaberscek, J. Power Sources, 2009, 189, 51.

21 K. J. Randall, W. Eberhardt, J. Feldhaus, W. Erlebach, A. M. Bradsham, Z. Xu, P. D. Johnson and Y. Ma, Nucl. Instrum. Methods Phys. Res., Sect. A, 1992, 319, 101-105.

22 J. Nordgren and J.-E. Rubensson, J. Electron Spectrosc. Relat. Phenom., 2013, 188, 3-9.

23 J. Nordgren, G. Bray, S. Cramm, R. Nyholm, J.-E. Rubensson and N. Wassdahl, Rev. Sci. Instrum., 1989, 60, 1690.

24 S. M. Butorin, J.-H. Guo, M. Magnuson, P. Kuiper and J. Nordgren, Phys. Rev. B: Condens. Matter Mater. Phys., 1996, 54, 4405.

25 B. Gilbert, B. H. Frazer, A. Belz, P. G. Conrad, K. H. Nealson, D. Haskel, J. C. Lang, G. Srajer and G. De Stasio, J. Phys. Chem. A, 2003, 107, 2839.
26 M. Dahbi, S. Urbonaita and T. Gustafsson, J. Power Sources, 2012, 205, 456-462.

27 T. Gustafsson, J. O. Thomas, R. Koksbang and G. C. Farrington, Electrochim. Acta, 1992, 37, 1639.

28 J. Rodriguez-Carvajal, Fullprof, Program for Rietveld Refinement, version 3.7, LLB JRC, 1997.

29 R. Dominko, M. Bele, M. Gaberscek, A. Meden, M. Remskar and J. Jamnik, Electrochem. Commun., 2006, 8, 217-222.

30 G. Mali, A. Meden and R. Dominko, Chem. Commun., 2010, 46, 3306-3308.

31 A. R. Armstrong, C. Lyness, M. Menetrier and P. G. Bruce, Chem. Mater., 2010, 22, 1892-1900.

32 H. M. Hollmark, T. Gustafsson, K. Edström and L.-C. Duda, Phys. Chem. Chem. Phys., 2011, 13, 20215. 